\title{
Erratum: Rigorous calculation of heating in alkali-metal traps by background gas collisions [Phys. Rev. A 61, 033606 (2000)]
}

\author{
H. C. W. Beijerinck \\ (Published 16 March 2001) \\ DOI: 10.1103/PhysRevA.63.049901 \\ PACS number(s): 03.75.Fi, 34.50.-s, 39.90.+d, 99.10.+g
}

The numerical values in Table I of the original paper have erroneously been calculated with the incorrect expression $v_{m}$ $=\left(3 k_{B} T_{b} / 2 m\right)^{1 / 2}$. In Table I of this erratum the correct expression $v_{m}=\left(2 k_{B} T_{b} / m\right)^{1 / 2}$ has been used, which results in new values for the parameters $k, Q, \sigma(0), \theta_{0}$, and the derived expressions $n_{b} \tau_{1}(1-\Delta Q / Q), \mathcal{E}_{\text {ref }}$, and $v_{m} Q \mathcal{E}_{\text {ref }}$.

The influence of these changes on the most important conclusion of the paper, i.e., the comparison with the trap heating data for Cs of the group of Chu [1], is rather small due to compensating effects. The correct value for the calculated energy transfer rate is $\dot{U}_{m} /\left(N k_{B}\right)=4.3 \mu \mathrm{K} / \mathrm{s}$, as compared to the experimental value of $\approx 4 \mu \mathrm{K} / \mathrm{s}$. The incorrect calculated result was $\dot{U}_{m} /\left(N k_{B}\right)=4.5 \mu \mathrm{K} / \mathrm{s}$. Although the calculated values in the other examples discussed in Sec. V of the paper also change slightly, the general insight remains without dispute.

TABLE I. System parameters for the alkali-metal gases, together with collision parameters $k, Q, \sigma(0)$, and $\theta_{0}$ at a velocity $v_{m}$ $=\left(2 k_{B} T_{b} / m\right)^{1 / 2}$ corresponding to the maximum of the Maxwell-Boltzmann distribution of the background gas atoms at temperature $T_{b}$ $=300 \mathrm{~K}$.

\begin{tabular}{|c|c|c|c|c|c|}
\hline Alkali & $\mathrm{Li}$ & $\mathrm{Na}$ & $\mathrm{K}$ & $\mathrm{Rb}$ & Cs \\
\hline$m$ (a.u.) & 7 & 23 & 39 & 87 & 133 \\
\hline$C_{6}(\text { a.u. })^{\mathrm{a}}$ & 1393 & 1556 & 3897 & 4691 & 6851 \\
\hline$v_{m}(\mathrm{~m} / \mathrm{s})$ & 844 & 466 & 358 & 239 & 194 \\
\hline$k\left(\AA^{-1}\right)$ & 4.67 & 8.47 & 11.0 & 16.5 & 20.4 \\
\hline$Q\left(\AA^{2}\right)$ & 951 & 1261 & 2023 & 2558 & 3240 \\
\hline$\sigma(0)\left(\AA^{2}\right)$ & $1.91 \times 10^{5}$ & $1.10 \times 10^{6}$ & $4.81 \times 10^{6}$ & $1.70 \times 10^{7}$ & $4.21 \times 10^{7}$ \\
\hline$\theta_{0}(\mathrm{mrad})$ & 24.6 & 11.8 & 7.1 & 4.3 & 3.1 \\
\hline$n_{b} \tau_{1}(1-\Delta Q / Q)\left(\mathrm{m}^{-3} \mathrm{~s}\right)$ & $1.25 \times 10^{14}$ & $1.70 \times 10^{14}$ & $1.38 \times 10^{14}$ & $1.63 \times 10^{14}$ & $1.59 \times 10^{14}$ \\
\hline $\mathcal{E}_{\text {ref }}(\mathrm{mK})$ & 45.4 & 10.4 & 3.83 & 1.36 & 0.702 \\
\hline$v_{m} Q \mathcal{E}_{r e f}\left(\mathrm{~m}^{3} \mathrm{~K} / \mathrm{s}\right)$ & $3.65 \times 10^{-16}$ & $6.12 \times 10^{-17}$ & $2.77 \times 10^{-17}$ & $8.32 \times 10^{-18}$ & $4.40 \times 10^{-18}$ \\
\hline
\end{tabular}

${ }^{\mathrm{a}}$ Reference [2].

[1] V. Vuletic', C. Chin, J. Kerman, and S. Chu, Phys. Rev. Lett. 81, 5768 (1998).

[2] A. Derevianko, W. Johnson, M. Safronova, and J. Babb, Phys. Rev. Lett. 82, 3589 (1999). 